\title{
Prevalence of Refractive Error and Visual Acuity Among School Children in the Plateau Region of Qinghai, China
}

\author{
Qiuxin $\mathrm{Wu}^{\mathrm{I}-3, *}$ \\ Qingmei Tian ${ }^{1-3, *}$ \\ Xiuyan Zhang ${ }^{1-3, *}$ \\ Jing $X u^{2}$ \\ Guodong Tang ${ }^{2}$ \\ Runkuan $\mathrm{Li}^{1}$ \\ Xiaoxiao Guo' \\ Zongqing $\mathrm{Xu}{ }^{\prime}$ \\ Jiaojiao Feng ' \\ Jike Song ${ }^{1-4}$ \\ Hongsheng $\mathrm{Bi}{ }^{1-4}$ \\ 'Shandong University of Traditional Chinese \\ Medicine, Jinan, 2500I4, People's Republic of \\ China; ${ }^{2}$ Affiliated Eye Hospital of Shandong \\ University of Traditional Chinese Medicine, \\ Jinan, 250002, People's Republic of China; \\ ${ }^{3}$ Shandong Provincial Key Laboratory of \\ Integrated Traditional Chinese and Western \\ Medicine for Prevention and Therapy of \\ Ocular Diseases, Key Laboratory of \\ Integrated Traditional Chinese and Western \\ Medicine for Prevention and Therapy of \\ Ocular Diseases in Universities of Shandong, \\ Eye Institute of Shandong University of \\ Traditional Chinese Medicine, Jinan, 250002, \\ People's Republic of China; ${ }^{4}$ Shandong \\ Provincial Key Laboratory of Integrative \\ Medicine for Eye Diseases, Shandong \\ Provincial Clinical Research Center of \\ Ophthalmology and Children Visual \\ Impairment Prevention and Control, \\ Shandong Engineering Technology Research \\ Center of Visual Intelligence, Shandong \\ Institute of Children Health and Myopia \\ Prevention and Control, Jinan, 250002, \\ People's Republic of China \\ *These authors contributed equally to this \\ work
}

Correspondence: Jike Song; Hongsheng Bi Tel +86-53I-58859696

Fax +86-53I-82432074

Email edusjk@I63.com;

hongshengbil@l63.com
Purpose: This study aimed to investigate the prevalence of refractive error and visual acuity among school children in the plateau region of Qinghai, China.

Methods: The school-based, cross-sectional study was performed in Menyuan, Qinghai, China. Three kindergartens and three primary schools were randomly enrolled from both rural areas and county towns. The participants had undergone ophthalmic examinations of the intraocular pressure (IOP), uncorrected visual acuity (UCVA), presenting visual acuity (PVA) and best corrected visual acuity (BCVA), cycloplegic refraction, and axial length (AL). Regression analysis was applied to investigate the potential risk factors affecting the prevalence of various refractive errors.

Results: A total of 3770 children were invited to participate, and 3524 (93.5\%) had undergone examination. Among the 3524 children ( $51.8 \%$ boys) with a mean age of $8.3 \pm 2.3$ years, 1049 (29.8\%) had myopia, $30(0.9 \%)$ had high myopia, 1692 (48.0\%) had mild hyperopia, $152(4.3 \%)$ had medium to marked hyperopia and $925(26.2 \%)$ had astigmatism. The mean SER was -0.16 $\pm 1.86 \mathrm{D}$ and decreased with age. The AL increased with age from $21.80 \pm 0.59 \mathrm{~mm}$ at 4 -years to $23.53 \pm 1.05 \mathrm{~mm}$ at 12 -years. The myopia prevalence increased with age from $2.0 \%$ at 4 years to $62.8 \%$ at 12 -years. Myopia was associated with increasing age, county town habitation and girls. Among the 723 participants with PVA 20/40 or worse in one eye, 564 (78.0\%) were due to uncorrected refractive error, and $83(22.0 \%)$ were due to undercorrected refractive error. Among the 1049 children with myopia, only 254 wore glasses, and 151 children with PVA had a worse BCVA and did not have accurate spectacles.

Conclusion: The prevalence of myopia is very high among school children in Menyuan. Only $24.2 \%$ of myopic children wore glasses, and $59.4 \%$ of children did not have accurate spectacles. Strategies to improve access to eye care and affordable glasses are needed.

Keywords: school children, refractive error, myopia, plateau region, glass wear

\section{Introduction}

Refractive errors such as hyperopia, myopia and astigmatism are the most general ocular disorders requiring spectacles or contact lenses for optimal vision. Refractive error is caused by the mismatch between the various optical elements of the eye, and the eye's axial length is one reason. ${ }^{1}$ Uncorrected errors of refraction are the most common causes of visual disability worldwide. ${ }^{2}$ The prevalence of refractive error varies with sex, geographic location and age. ${ }^{3-6}$

Myopia is a widespread refractive error among children and teenagers and is globally recognized as a major twenty-first century public health problem. ${ }^{7,8}$ Dong 
et al speculated that the myopia prevalence in 2050 among Chinese children and adolescents aged 3 to 19 years will be approximately $84 \% .{ }^{9}$ Myopia causes further vision challenges because high myopia increases the risk of severe and irreversible vision loss, such as cataracts, glaucoma, retinal detachment, and myopic macular degeneration. ${ }^{10}$ Thus, preventing the occurrence and development of myopia in children and adolescents is crucial.

Myopia is associated with genetic, behavioral, social and environmental factors, including socioeconomic status, educational attainment, ${ }^{11}$ time spent outdoors, ${ }^{12}$ outdoor light intensity ${ }^{13}$ and time spent reading. ${ }^{14}$ Although previous studies have provided valuable insights into the etiology of myopia, natural environmental factors have largely been neglected. Many reports have investigated the prevalence of myopia in China's plains, ${ }^{15-18}$ but few reports are available on the prevalence of myopia in the northwest plateau.

The Qinghai-Tibet Plateau is the largest plateau worldwide. This area is characterized by low temperature, large temperature differences between day and night, long sunshine, strong solar radiation, high altitude, and thin oxygen. The high altitude and special climate on the Qinghai-Tibet Plateau can affect people's physical and mental health, such as hypertension, ${ }^{19}$ cardiovascular diseases, ${ }^{20}$ cognitive impairments ${ }^{21}$ and depression. ${ }^{22}$ These specific geographical environments also affect eye health, ${ }^{23}$ including pterygium, cataracts, and dry eye syndrome. Few reports have examined the prevalence of myopia among children on the Qinghai-Tibet Plateau. Only 3 previous reports on Qinghai-Tibet Plateau school children are available to date. The first study reported that from 2008 to 2009 , the prevalence of myopia was $11.8 \%$ in primary school students in Qinghai. ${ }^{24}$ The second study reported that in 2014, among children and adolescents aged 7 to 18 years, the prevalence of myopia was $48.2 \%$ in Qinghai and $62.9 \%$ in Tibet. $^{25}$ The third study reported that in 2018, the prevalence of myopia in Tibet was $23.83 \%$ among children, with a mean age of 12.69 $( \pm 2.88)$ years. $^{26}$

No epidemiological study has been performed on refractive error in school children in Qinghai in the past 5 years. We selected Menyuan Hui Autonomous County (Menyuan) in Qinghai Province as the study site. Through ocular examination, we analyzed the refractive status and vision acuity in Qinghai Province.

\section{Materials and Methods}

\section{Ethics Statement}

This study was approved by the ethics committee of the Affiliated Eye Hospital of Shandong University of Traditional Chinese Medicine and Administration of the Education and School Board of Menyuan. This study adhered to the tenets of the Declaration of Helsinki. Additionally, we obtained written informed consent from at least one parent or legal guardian of each participant.

\section{Recruitment}

The pediatric eye study was a school-based, crosssectional study of children in the northwest Chinese province of Qinghai conducted in Menyuan in September 2019. The sampling frame was based on 11 kindergartens and 12 primary schools with approximately 18,381 children throughout Menyuan, excluding special schools for the disabled. Three kindergartens and 3 primary schools from both rural areas and county towns were selected randomly.

\section{Ophthalmic Examination}

The children had undergone the following examinations: distance visual acuity (VA) testing, intraocular pressure (IOP), cover test, slit-lamp examination, fund examination, cycloplegic autorefraction, and axial length (AL).

Before the VA test, the children were asked whether they wore glasses, contact lenses or orthokeratology contact lenses. For the children not wearing glasses, the uncorrected visual acuity (UCVA) and best corrected visual acuity (BCVA) were tested for both eyes. For those wearing glasses, presenting visual acuity (PVA) was first measured, and then UCVA was measured after removing the glasses for 30 minutes. BCVA was examined by subjective refraction by experienced optometrists. VA was measured using a tumbling "E" chart (\#600722; Good-Lite Co., Elgin, IL, USA) (the VA chart was 3 meters away from the child. The lowest line of the chart was approximately as high as the eye of the child. The children were asked to indicate the direction of the "E" opening starting on the first line of the chart (VA: 20/100) and moving on to the next line if none or one character of the line was incorrect. When the child incorrectly described at least 2 characters, VA was recorded as the value of the previous line. If the children could not read 20/100 lines at a distance of 3 meters, the test was repeated at one meter. If a single line could not be read at this 
distance, vision was assessed by counting fingers, hand movement or light perception.

The purpose of IOP measurement and slit-lamp examination was to exclude contraindications for mydriasis. Cycloplegia was achieved after instilling one drop of topical anesthetic ((Minims Oxybuprocaine Hydrochloride 0.4\% w/v; Santen Pharmaceutical Co., Ltd; Japan) and one drop of cyclopentolate hydrochloride (Minims; 1\% w/v; Alcon Laboratories, Inc; USA). The children were administered two drops of cyclopentolate hydrochloride 5 min apart. Once cycloplegia had been established, at least $20 \mathrm{~min}$ after instilling the eye drops, autorefraction was performed. Full cycloplegia was considered when the pupil diameter reached $6 \mathrm{~mm}$ or more and the light reflex disappeared. The presence of refraction with cycloplegia was assessed using an autorefractor (ARK-1; NIDEK, Tokyo, Japan). AL was measured using an IOL Master500 (Carl Zeiss Meditec AG, Jena, Germany).

All the above instruments were calibrated before the ocular examination. All the examinations were performed by board-certified ophthalmologists and certified optometrists.

\section{Definitions}

Refraction measurements were converted into spherical equivalent refraction (SER), which was calculated as the spherical value plus half of the astigmatism value. Medium to marked hyperopia was defined as $>+2.0$ dioptres (D), and mild hyperopia was defined as $>+0.50 \mathrm{D}$ and $\leq+2.0 \mathrm{D}$, in one or both eyes if neither eye was myopic. Emmetropia was defined as $>-0.50 \mathrm{D}$ and $\leq+0.50 \mathrm{D}$, in both eyes. Myopia was defined as $\leq-0.5 \mathrm{D}$ and high myopia as $\leq-6.0 \mathrm{D}$, in one or both eyes. Astigmatism was defined as a myopic cylindrical refractive error $\leq-0.75$ $\mathrm{D}$ in either eye. All refractive errors were measured under cycloplegia.

For the students not wearing glasses, PVA is equivalent to UCVA; for those wearing glasses, PVA is equivalent to VA of wearing spectacles.

\section{Statistical Methodology}

Statistical analyses were performed using SPSS 19.0 (SPSS Inc., Chicago, IL). For normally distributed continuous variables, the data were expressed as means \pm standard deviation (SD). Categorical variables were expressed as frequencies of the total. The risk factors associated with myopia were determined by two-level multivariate logistic regression analysis. First, we performed univariate binary logistic regression analysis to evaluate the potential association. Multivariate logistic regression was then performed to analyze all statistically significant factors found in the univariate analysis. For continuous variables, we conducted univariate analysis to identify correlations and then performed linear regression analysis. In our results, odds ratios (ORs) and $95 \%$ confidence intervals (CIs) were calculated to evaluate myopia risk factors. $\mathrm{OR}<1.0$ and $P<0.05$ demonstrated that a parameter was a protective factor, while $\mathrm{OR}>1.0$ and $P<0.05$ demonstrated that a parameter was a risky factor. Statistical tests were two-sided, and $P<0.05$ was considered statistically significant.

\section{Results}

\section{Participant Characteristics}

During September 2019, 3770 participants were recruited. Of these, $233(6.18 \%)$ children refuse to participate and not signed informed consent, $13(0.34 \%)$ with an IOP $\geq 25$ $\mathrm{mmHg}$ in one or both eyes were excluded from the study, and 3524 completed the ophthalmic examinations, resulting in a complete examination rate of $93.47 \%$. The demographic characteristics of the study population are described in Table 1.

The mean age of the 3524 participants was $8.3 \pm 2.3$ years (4-12 years). Of the 3524 participants, 1798 (51.0\%) resided in rural areas, and $1726(49.0 \%)$ resided in county towns. The participants comprised more boys (1825; $51.8 \%)$ than girls $(1699 ; 48.2 \%)$ among, but no significant difference was found in the sex between participants in rural areas (53.4\% boys) and county towns (50.1\% boys) $\left(\chi^{2}=3.787 ; P=0.052\right)$.

Table I The Demographic Characteristics of the Study Participants

\begin{tabular}{|l|c|c|c|}
\hline $\begin{array}{l}\text { Age } \\
\text { (Years) }\end{array}$ & $\begin{array}{c}\text { Number (\%) } \\
\text { of Invited } \\
\text { Population }\end{array}$ & $\begin{array}{c}\text { Number (\%) of } \\
\text { Completed } \\
\text { Examinations }\end{array}$ & $\begin{array}{c}\text { Percentage of } \\
\text { Completed } \\
\text { Examinations(\%) }\end{array}$ \\
\hline 4 & $239(6.3)$ & $201(5.7)$ & 84.1 \\
5 & $352(9.3)$ & $316(9.0)$ & 89.8 \\
6 & $356(9.4)$ & $324(9.2)$ & 91.0 \\
7 & $468(12.4)$ & $438(12.4)$ & 93.6 \\
8 & $520(13.8)$ & $490(13.9)$ & 94.2 \\
9 & $532(14.1)$ & $504(14.3)$ & 94.7 \\
10 & $499(13.2)$ & $480(13.6)$ & 96.2 \\
11 & $511(13.6)$ & $483(13.7)$ & 94.5 \\
12 & $293(7.8)$ & $288(8.2)$ & 98.3 \\
Total & $3770(100.0)$ & $3524(100.0)$ & 93.5 \\
\hline
\end{tabular}



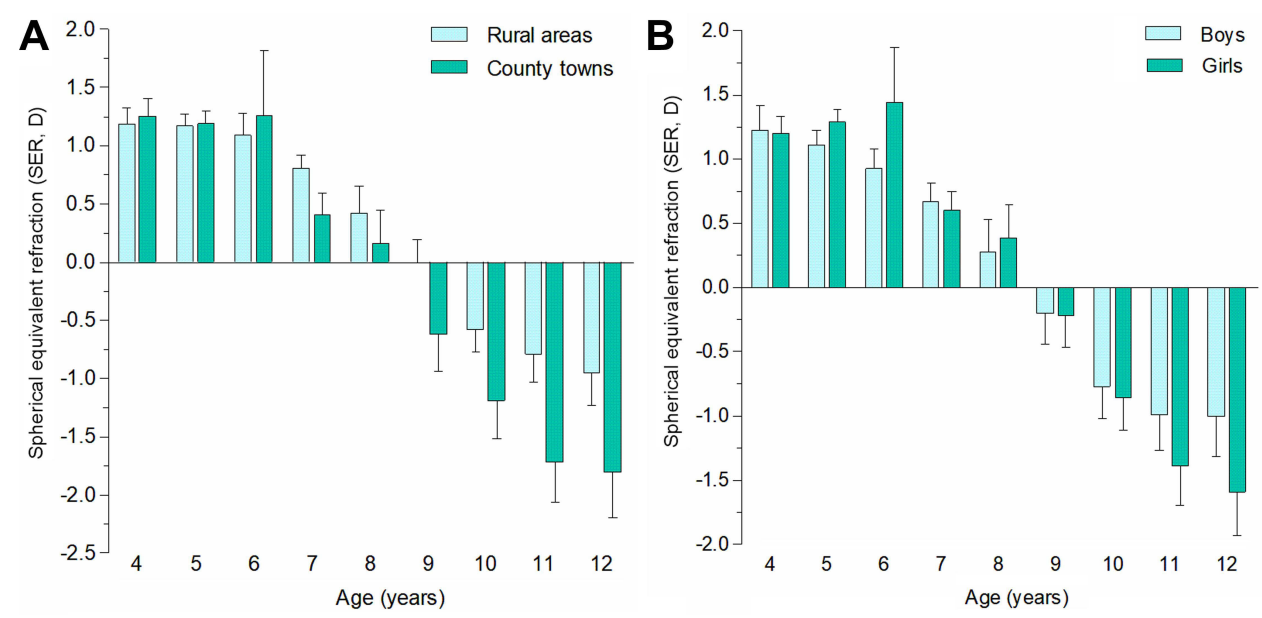

Figure I Spherical equivalent (SE, D) distribution of right eyes, (A) stratified by age and region of habitation, (B) stratified by age and genders in the Qinghai children. The $Y$ axis shows the mean and $95 \%$ confidence interval.

\section{Ocular Parameters}

A significant difference was found in SER between the right and left eyes ( $\mathrm{t}=2.071 ; P=0.038)$. The mean SER of the right eyes was $-0.16 \pm 1.86 \mathrm{D}$ (median: $0.38 \mathrm{D}$; range: $-16.5 \mathrm{D}$ to $+11.75 \mathrm{D}$ ) and that of the left eyes was $-0.05 \pm 1.80 \mathrm{D}$ (median: $0.38 \mathrm{D}$; range: $-13.25 \mathrm{D}$ to $+12.00 \mathrm{D}$ ). Pearson correlation analysis of the SER showed a high correlation $(\mathrm{r}=0.914$; $P=0.000$ ) between the right and left eyes. Therefore, only the SER of the right eyes was analyzed in our study.

The mean SER of children in the county towns $(-0.44$ $\pm 2.08 \mathrm{D})$ showed a greater refractive change toward myopia than that of children in rural areas $(0.02 \pm 1.68 \mathrm{D})(\mathrm{t}=-5.983$; $P=0.000$ ). The SER decreased within children aged 4 to 12 years in county towns $(\mathrm{F}=47.385 ; P=0.000)$ and rural areas $(\mathrm{F}=44.944 ; P=0.000)$ (Figure 1A). In the county towns, the SERs were $1.25 \pm 0.59,1.19 \pm 0.55,1.26 \pm 1.51,0.41 \pm 0.97$, $0.16 \pm 1.77,-0.62 \pm 1.91,-1.19 \pm 2.09,-1.72 \pm 2.34$ and -1.80 $\pm 1.96 \mathrm{D}$ in children aged 4 to 12 years. In the rural areas, the SERs were $1.18 \pm 0.55,1.17 \pm 0.55,1.09 \pm 0.90,0.81 \pm 0.72$, $0.42 \pm 1.83,0 \pm 1.55,-0.58 \pm 1.60,-0.79 \pm 1.87$ and -0.95 $\pm 1.72 \mathrm{D}$ in children aged 4 to 12 years.

The SER was $-0.09 \pm 1.79 \mathrm{D}$ in boys and $-0.24 \pm 1.94$ $\mathrm{D}$ in girls, and the difference was statistically significant $(\mathrm{t}=2.040 ; P=0.041)$. The SER decreased with age in both boys and girls but was more pronounced in girls (Figure 1B). In boys, the SERs were $1.22 \pm 0.68,1.11$ $\pm 0.59, \quad 0.93 \pm 0.64, \quad 0.67 \pm 0.85, \quad 0.28 \pm 1.83, \quad-0.2 \pm 1.69$, $-0.77 \pm 1.8,-0.99 \pm 2.11$ and $-1 \pm 1.78 \mathrm{D}$ in those aged 4 to 12 years. In girls, the SERs were $1.201 \pm 0.46,1.29 \pm 0.45$, $1.44 \pm 1.49,0.6 \pm 0.86,0.39 \pm 1.81,-0.22 \pm 1.73,-0.86 \pm 1.86$, $-1.39 \pm 2.12$ and $-1.59 \pm 1.9 \mathrm{D}$ in those aged 4 to 12 years.
The AL was significantly longer with each older age, from $21.80 \pm 0.59 \mathrm{~mm}$ in 4-year-olds to $23.53 \pm 1.05 \mathrm{~mm}$ in 12-year-olds ( $\mathrm{F}=98.006 ; P=0.000)$. The $\mathrm{AL}$ of children in county towns $(23.25 \pm 1.20 \mathrm{~mm})$ was longer than that in rural areas $(22.81 \pm 1.03 \mathrm{~mm})(\mathrm{t}=-7.156 ; P=0.000)$ (Figure 2A). In the county towns, the ALs were $21.8 \pm 0.6,21.93 \pm 0.72$, $22.37 \pm 0.93,22.72 \pm 0.72, \quad 22.84 \pm 1.02, \quad 23.36 \pm 0.96, \quad 23.62$ $\pm 1.10,23.92 \pm 1.13$ and $23.83 \pm 1.21 \mathrm{~mm}$ in children aged 4 to 12 years. In the rural areas, the ALs were $21.81 \pm 0.59$, $22.01 \pm 0.7, \quad 22.26 \pm 0.69, \quad 22.33 \pm 0.84, \quad 22.63 \pm 1.04, \quad 22.84$ $\pm 0.96,23.19 \pm 0.98,23.32 \pm 1.02$ and $23.34 \pm 0.89 \mathrm{~mm}$ in children aged 4 to 12 years.

The difference in the AL between boys (23.16 $\pm 1.07 \mathrm{~mm})$ and girls $(22.70 \pm 1.11 \mathrm{~mm})$ was statistically significant $(\mathrm{t}=10.930 ; P=0.000)$ (Figure $2 \mathrm{~B})$. In boys, the ALs were $22.09 \pm 0.61,22.25 \pm 0.57,22.58 \pm 0.64,22.77$ $\pm 0.77,22.93 \pm 0.95,23.28 \pm 0.96,23.66 \pm 1.00,23.74 \pm 1.09$ and $23.61 \pm 1.10 \mathrm{~mm}$ in those aged 4 to 12 years. In girls, the ALs were $21.59 \pm 0.48,21.58 \pm 0.71,21.88 \pm 0.73,22.19$ $\pm 0.74,22.46 \pm 1.08,22.76 \pm 0.96,23.07 \pm 1.01,23.34 \pm 1.08$ and $23.44 \pm 0.99 \mathrm{~mm}$ in those aged 4 to 12 years.

\section{Prevalence of Refractive Errors}

The prevalence of myopia was $29.8 \%$ (95\% CI: $20.7 \%$, $39.0 \%$ ) in the 3524 children (Table 2). The prevalence of myopia increased with age from $2.0 \%(95 \% \mathrm{CI}:-1.7 \%$, $6.2 \%)$ in 4-year-olds to $62.8 \%$ (95\% CI: $42.6 \%, 83.6 \%)$ in 12-year-olds (Table 3 and Figure 3). Univariate binary logistic regression analysis demonstrated that the prevalence of myopia was significantly associated with girls ( $P=0.043)$, increasing age $(P=0.000)$ and habitation in 

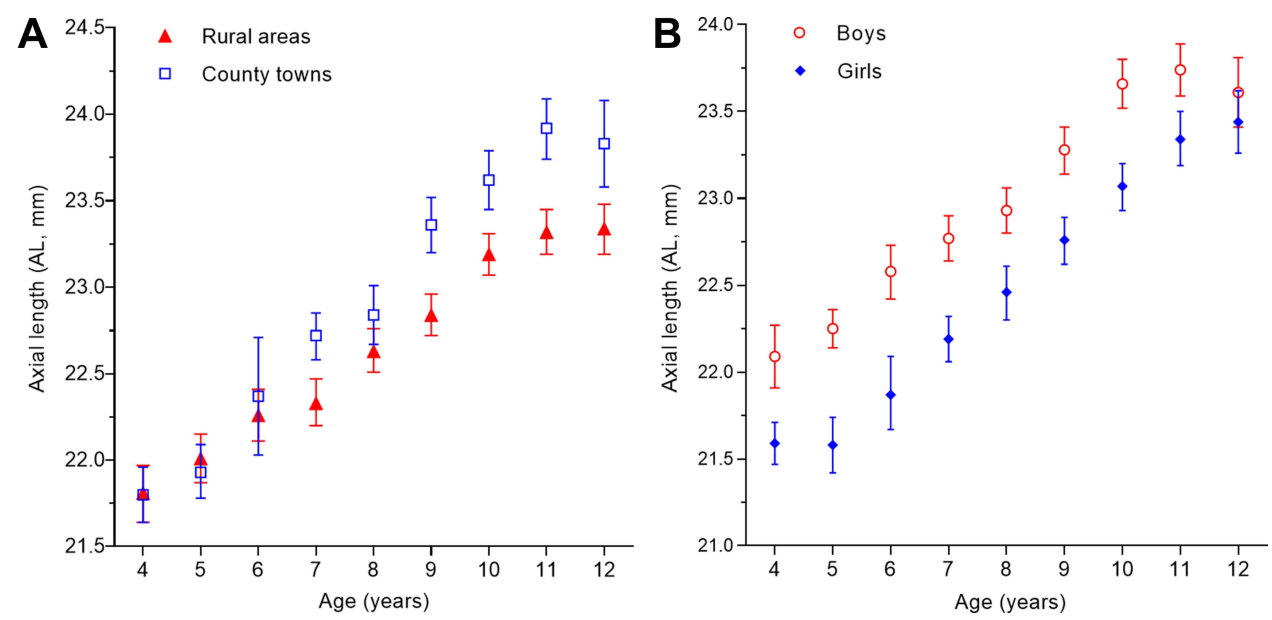

Figure 2 Axial length (AL, mm) distribution of right eyes, (A) stratified by age and region of habitation, (B) stratified by age and genders in the Qinghai children. The $\mathrm{Y}$ axis shows the mean and $95 \%$ confidence interval.

county towns ( $P=0.000)$. The results of multivariate binary regression analysis revealed that the prevalence of myopia was higher in female students than in male students, and female students had a 1.22-fold higher risk of myopia than male students [OR: 1.22 (95\% CI: 1.03, 1.45); $P=0.043$ ] The results also indicated that the prevalence of myopia increased with age [OR: 1.84 (95\% CI: 1.75, 1.93); $P=0.000]$. Furthermore, children living in county towns [OR: 1.53 (95\% CI: $1.29,1.80) ; P=0.000]$ had a higher risk of myopia than those living in rural areas.

The prevalence of high myopia was $0.9 \%$ (Table 2 ) in total and increased from $0 \%$ in the 4 -year-old group to $2.1 \%$ in the 12 -year-old group (Table 3). In univariate binary logistic regression analysis, the prevalence of high myopia was significantly associated with increasing age $(P=0.000)$, but it was not significantly associated with region of habitation $(P=0.229)$ or sex $(P=0.191)$. As the children became older, their risk of high myopia increased [OR: 1.67 (95\% CI: 1.33, 2.09); $P=0.000]$.

The prevalence of mild hyperopia was $48.0 \%$ in total (Table 2) and decreased from $89.1 \%$ in the 4 -year-old group to $16.7 \%$ in the 12 -year-old group (Table 3). Univariate binary logistic regression analysis showed that the prevalence of mild hyperopia was significantly associated with increasing age $(P=0.000)$, but it was not significantly associated with region of habitation $(P=0.512)$ or sex $(P=0.970)$. As the children became older, their risk of mild hyperopia decreased (OR: 0.60 [95\% CI: 0.57, $0.62] ; P=0.000$ ).

The prevalence of medium to marked hyperopia was $4.3 \%$ in total (Table 2) and decreased from $5 \%$ in the 4-year-old group to $1.0 \%$ in the 12-year-old group
(Table 3). Univariate analysis showed that the prevalence of medium to marked hyperopia was significantly associated with increasing age $(P=0.001)$, but was not associated with region of habitation $(P=0.672)$ or sex ( $P=0.405)$. As children became older, their risk of medium to marked hyperopia decreased (OR: 0.89 [95\% CI: 0.83 , 0.95]; $P=0.001$ ).

The prevalence of astigmatism was $26.2 \%$ in total (Table 2), and the mean value of astigmatism was 0.43 $\pm 0.51 \mathrm{D}$ (median: $-0.25 \mathrm{D}$; range: $-7.00 \mathrm{D}$ to $0 \mathrm{D}$ ). Univariate analysis showed that the prevalence of astigmatism was significantly associated with age [regression coefficient $\mathrm{B}=-0.02$; standardized coefficients beta $=$ -0.10 ; $P=0.000$; (95\% CI: $-0.03,-0.02)$ ], region of habitation $(P=0.009)$, sex $(P=0.033)$ and myopic refractive error [regression coefficient $\mathrm{B}=0.02$; standardized coefficients beta $=0.07 ; P=0.000 ;(95 \%$ CI: $0.01,0.03)]$. In binary regression analysis, older age [OR: $1.09(95 \%$ CI: $1.04,1.13) ; P=0.000]$, habitation in county towns [OR: 1.19 (95\% CI: 1.02, 1.39); $P=0.000]$, and more myopic refractive error $[\mathrm{B}=-1.06$; OR: $0.90(95 \% \mathrm{CI}$ : $0.86,0.94) ; P=0.000]$ were associated with a higher astigmatism prevalence. Girls [OR: 0.85 (95\% CI: 0.73, 0.99); $P=0.035]$ were associated with a lower prevalence of astigmatism.

\section{Visual Acuity and Glasses Wear}

In high myopia, myopia and medium to marked hyperopia, the uncorrected visual acuity (UCVA) and presenting visual acuity (PVA) were significantly lower than those in emmetropia, while no difference was found in UCVA $(P=0.105)$ and PVA $(P=0.193)$ between mild hyperopia 


\begin{tabular}{|c|c|c|c|c|c|}
\hline \multirow{2}{*}{ 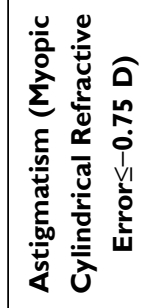 } & 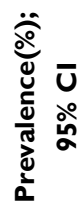 & 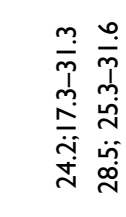 & $\begin{array}{l}\tilde{N} \\
\tilde{N} \\
0 \\
0 \\
\underline{0} \\
\dot{+} \\
\dot{d}\end{array}$ & $\begin{array}{l}\bar{i} \\
\tilde{N} \\
\tilde{m} \\
\stackrel{\sim}{\sim} \\
\dot{\sim} \\
\stackrel{\sim}{\infty}\end{array}$ & 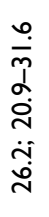 \\
\hline & $\mathbf{z}$ & 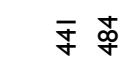 & $\stackrel{\infty}{\%}$ & $\stackrel{\hat{\infty}}{\not{q}}$ & $\stackrel{\sim}{\sigma}$ \\
\hline \multirow{2}{*}{ 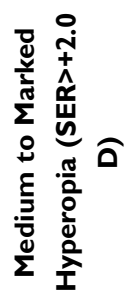 } & 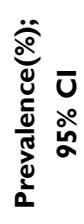 & 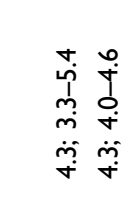 & $\begin{array}{l}\infty \\
\dot{j} \\
0 \\
\dot{j} \\
\dot{+} \\
\dot{y}\end{array}$ & $\begin{array}{l}\stackrel{n}{p} \\
\hat{n} \\
\ddot{n} \\
\dot{r}\end{array}$ & $\begin{array}{l}\hat{y} \\
\dot{y} \\
\dot{m} \\
\dot{m} \\
\dot{v}\end{array}$ \\
\hline & $\mathbf{z}$ & $2 \Re$ & $\stackrel{n}{\wedge}$ & 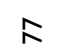 & 드 \\
\hline \multirow{2}{*}{ 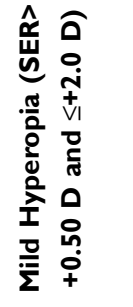 } & 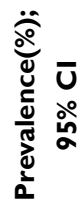 & 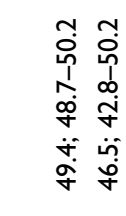 & $\begin{array}{l}m \\
0 \\
\hat{p} \\
\hat{j} \\
\dot{f} \\
\dot{0} \\
\dot{\alpha} \\
\dot{\alpha}\end{array}$ & 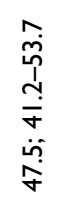 & 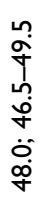 \\
\hline & z & ธ్ ః & $\underset{\infty}{\stackrel{\infty}{\infty}}$ & $\frac{\sigma}{\infty}$ & 응 \\
\hline \multirow[t]{2}{*}{ 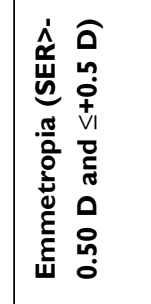 } & 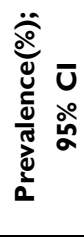 & 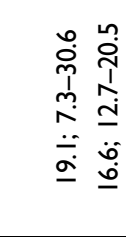 & 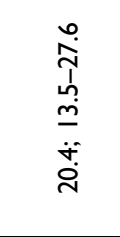 & 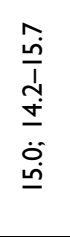 & 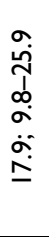 \\
\hline & $\mathbf{z}$ & 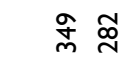 & $\underset{m}{\stackrel{m}{m}}$ & $\stackrel{\infty}{\sim}$ & $\overline{3}$ \\
\hline \multirow{2}{*}{ 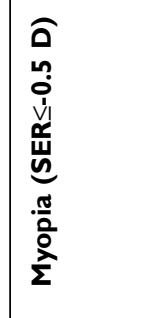 } & 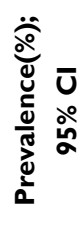 & 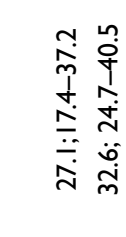 & 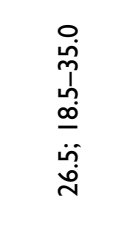 & 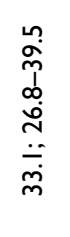 & 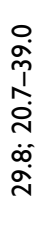 \\
\hline & $\mathbf{z}$ & 骂苟 & $\hat{f}$ & हn & 守 \\
\hline \multirow[t]{2}{*}{ 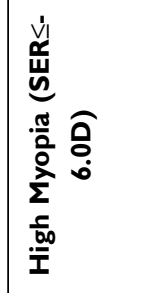 } & 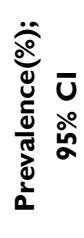 & 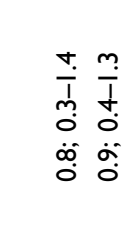 & $\begin{array}{l}\infty \\
0 \\
0 \\
0 \\
0 \\
\hat{0} \\
0\end{array}$ & $\begin{array}{l}\frac{0}{0} \\
\dot{d} \\
\dot{0}\end{array}$ & $\begin{array}{l}+ \\
\vdots \\
\vdots \\
0 \\
\dot{\alpha} \\
0 \\
0\end{array}$ \\
\hline & $z$ & $\underline{n} \underline{\underline{n}}$ & $\simeq$ & $\underline{\infty}$ & ㅇ \\
\hline \multicolumn{2}{|l|}{ z } & 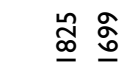 & 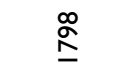 & $\stackrel{\stackrel{N}{I}}{=}$ & $\underset{\sim}{\stackrel{్}{n}}$ \\
\hline & & 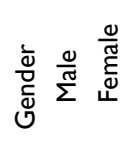 & 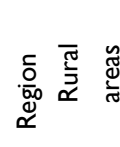 & 离 & \\
\hline
\end{tabular}

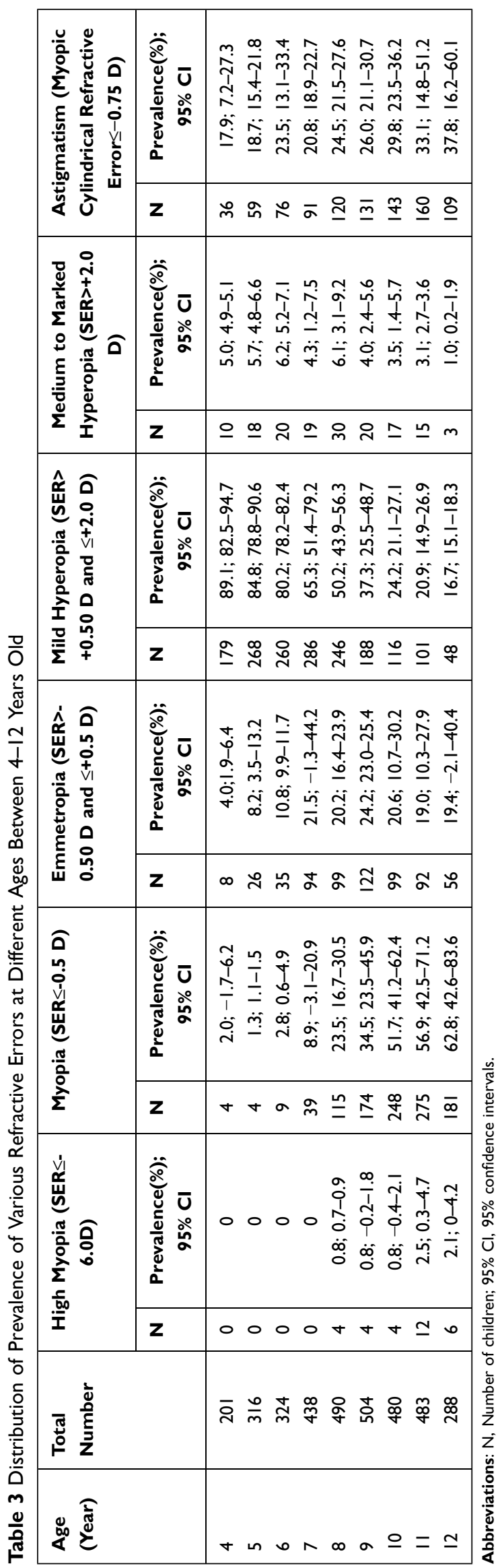




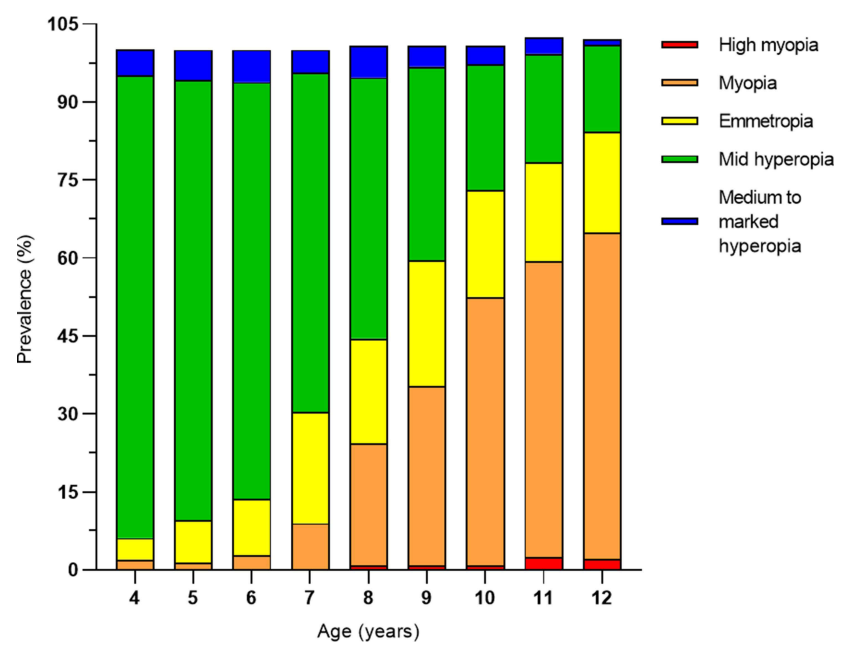

Figure 3 Prevalence of high myopia, myopia, emmetropia, mild hyperopia and medium to marked hyperopia, stratified by age in the Qinghai children. Participants were classified according to SER into high myopia (SER $\leq-6.0 D)$, myopia $(\mathrm{SER} \leq-0.5 \mathrm{D}$ ), emmetropia (SER>-0.50 D and $\leq+0.5 \mathrm{D}$ ), mild hyperopia (SER> $+0.50 \mathrm{D}$ and $\leq+2.0 \mathrm{D})$, and medium to marked hyperopia $(>+2.0 \mathrm{D})$.

and emmetropia. The best corrected visual acuity (BCVA) in high myopia $(P=0.000)$ and medium to marked hyperopia $(P=0.000)$ was significantly lower than that in emmetropia, but no difference was found in the BCVA in mild hyperopia $(P=0.866)$, myopia $(P=0.283)$ and emmetropia. High myopia and medium to marked hyperopia affected the children's vision.

The VA results are presented in Table 4. Refractive errors were the main reason for UCVA $20 / 40$ or worse. Of the 3524 participants, 2917 (82.8\%) had a UCVA of 20/30 or better in at least one eye, $607(17.2 \%)$ had a UCVA of $20 / 40$ or worse in the better eye, and $411(11.7 \%)$ had a PVA of $20 / 40$ or worse in the better eye. With best correction, VA 20/40 or worse in the better eye decreased to $40(1.1 \%)$. Among the 891 participants with UCVA 20/ 40 or worse in one eye, $812(91.1 \%)$ were caused by uncorrected refractive error. Among the 723 participants with PVA 20/40 or worse in one eye, 564 (78.0\%) were caused by uncorrected refractive error, and $83(22.0 \%)$ were caused by undercorrected refractive error.

All the participants were asked to bring their spectacles to the school on the day of the ocular examination. In the 3524 participants with reliable VA, glasses were only used by $288(8.2 \%)$. Two hundred fifty-four participants wore glasses due to myopia, which was the main reason for them to wear glasses. Additionally, among the 1049 myopic participants, 795 (75.8\%) participants presented without glasses. Furthermore, 302 (38.0\%) participants without glasses had a UCVA of 20/40 or worse in the better eye (Table 5). Among 254 (24.2\%) myopic participants who wore glasses, $44(17.3 \%)$ had a PVA $20 / 40$ or worse in the better eye, and 151 (59.4\%) presenting with PVA had a worse BCVA and did not have accurate spectacles.

\section{Discussion}

Menyuan is located on the high-altitude Qinghai-Tibet Plateau, and the environment is expected to affect the eye health of children living there, but little is known about the prevalence of refractive error in the northwest plateau region. Therefore, we investigated the characteristics of refractive error among school-aged children.

In our study, the boys living in the county town and with older age had longer eyes. Higher AL was related to older age of the children. ${ }^{27}$ In the current study, the $\mathrm{AL}$ became significantly longer with each older age from $21.80 \pm 0.59 \mathrm{~mm}$ in 4-year-olds to $23.53 \pm 1.05 \mathrm{~mm}$ in 12 year-olds. Male sex was a significant and independent factor for a longer axial length. ${ }^{28}$ In the present data, the $\mathrm{AL}$ was longer in boys than in girls, a finding that agrees with a previous study. ${ }^{28,29}$ The mean AL in our study was $23.61 \pm 1.10 \mathrm{~mm}$ for boys and $23.44 \pm 0.99 \mathrm{~mm}$ for girls at 12 years old. The values were slightly shorter than the ALs obtained in one Eastern Chinese study on children of the same age (boys: $24.14 \pm 1.01 \mathrm{~mm}$; girls: $23.69 \pm 1.10 \mathrm{~mm}$ ).

Table 4 The Uncorrected, Present and Best Corrected Visual Acuity (UCVA, PVA and BCVA) Distribution

\begin{tabular}{|l|c|c|c|c|c|c|}
\hline \multirow{2}{*}{ Visual Acuity Category } & \multicolumn{2}{|c|}{ UCVA } & \multicolumn{2}{c|}{ PVA } & \multicolumn{2}{c|}{ BCVA } \\
\cline { 2 - 7 } & N & Percentage (\%); 95\% CI & N & Percentage (\%); 95\% Cl & N & Percentage (\%); 95\% CI \\
\hline$\geq 20 / 30$ in both eyes & 2633 & $74.7 ; 63.6-85.8$ & 2800 & $79.5 ; 77.8-81.1$ & 3432 & $97.4 ; 97.1-97.7$ \\
$\geq 20 / 30$ in one eye & 284 & $8.1 ; 7.6-8.5$ & 313 & $8.9 ; 7.8-10.0$ & 52 & $1.5 ; 1.2-1.7$ \\
$20 / 40$ to 20/63 in better eye & 325 & $9.2 ; 6.6-11.9$ & 287 & $8.1 ; 7.8-8.5$ & 21 & $0.6 ; 0.5-0.7$ \\
$20 / 80$ to 20/160 in better eye & 202 & $5.7 ; 0-11.4$ & 94 & $2.7 ; 2.0-3.3$ & 15 & $0.4 ; 0-0.8$ \\
$\leq 20 / 200$ in better eye & 80 & $2.3 ; 0-4.6$ & 30 & $0.9 ; 0.4-1.3$ & 4 & $0.1 ; 0.1-0.1$ \\
Total & 3524 & 100 & 3524 & 100 & 3524 & 100 \\
\hline
\end{tabular}

Abbreviations: N, Number of children; $95 \% \mathrm{Cl}, 95 \%$ confidence intervals. 
Table 5 The Distribution of Presenting Visual Acuity (PVA) and Best Corrected Visual Acuity (BCVA) Among Myopic Participants

\begin{tabular}{|l|c|c|c|c|c|c|}
\hline \multirow{2}{*}{ Visual Acuity Category } & \multicolumn{2}{|c|}{$\begin{array}{c}\text { Myopia Yet Not Wearing Glasses } \\
\text { (UCVA=PVA) }\end{array}$} & \multicolumn{2}{|c|}{$\begin{array}{c}\text { Myopia Wearing Glasses } \\
\text { (PVA) }\end{array}$} & \multicolumn{2}{c|}{ BCVA } \\
\cline { 2 - 6 } & N & Percentage (\%) & N & Percentage (\%) & N & Percentage (\%) \\
\hline$\geq 20 / 30$ in both eyes & 332 & 31.6 & 167 & 15.9 & 1022 & 97.4 \\
$\geq 20 / 30$ in one eye & 161 & 15.3 & 43 & 4.1 & 13 & 1.2 \\
$20 / 40$ to 20/63 in better eye & 203 & 19.4 & 34 & 3.2 & 6 & 0.6 \\
$20 / 80$ to 20/160 in better eye & 76 & 7.2 & 8 & 0.8 & 7 & 0.7 \\
$\leq 20 / 200$ in better eye & 23 & 2.2 & 254 & 0.2 & 0 & 0 \\
Total & 795 & 75.8 & & 24.2 & 1049 & 1 \\
\hline
\end{tabular}

We found the same phenomenon as in previous studies ${ }^{29}$ and at the same age: boys have longer ALs and higher refractive power than girls; however, the reasons must be further clarified.

The prevalence of myopia among 4- to 12-year-old children in Qinghai was $29.8 \%$. Qian et $\mathrm{al}^{26}$ reported that the prevalence of myopia was $23.83 \%$ in Tibet (12.69 \pm 2.88 years old). Xie et $\mathrm{al}^{30}$ defined $\mathrm{VA}<20 / 20$ and $\mathrm{SER}<-0.5 \mathrm{D}$ as myopia and found that the myopia prevalence of children aged 7-13 years in Chongqing was $33.1 \%$. Wang et $\mathrm{al}^{16}$ defined myopia as SER $\leq-0.5 \mathrm{D}$ and UCVA $\leq 20 / 25$ and reported that the myopia prevalence was $41.4 \%$ in Yiwu among 5- to 12-year-old children. Both Qinghai and Tibet are economically backward plateau regions, and they have similar myopia prevalences. The prevalence of myopia in Qinghai is not significantly lower than that in Chongqing and Yiwu economically developed areas. Our study, together with previous studies in school children, suggests that myopia is a serious public health problem in China, including plateau regions. The government should be aware of these findings and adopt measures to prevent myopia among children in China's plateau regions.

Qinghai is characterized by low temperature, large temperature differences between day and night, long sunshine, and strong solar radiation, making children spend relatively less time in outdoor activities and increasing the possibility of myopia. Studies have shown that scleral hypoxia is one cause of myopia. ${ }^{31}$ The high altitude of Menyuan and low oxygen content in the air affect the oxygen supply of the eyes, a factor that may also affect myopia in children.

Myopia was significantly associated with increasing age, county town region of habitation and girls, findings that are consistent with previous study findings. ${ }^{3-5}$ The learning burden increases with age, leading to more hours on near work activities and reduced time on outdoor activities, a situation considered to be an important risk factor for the occurrence of myopia. ${ }^{32,33}$ The environment is an important factor in the prevalence of myopia, and county town children have a higher risk of myopia than rural area children subjected to the county town environment and learning pressure. ${ }^{3,17}$ Regarding sex differences, girls tend to spend more time reading and writing and perform less outdoor activities, making them more vulnerable to developing myopia. ${ }^{34}$

As children become older, their risk of high myopia increases. The prevalence of high myopia increased from $0.8 \%$ in 8 -year-old patients to $2.1 \%$ in 12 -year-old patients. In 12-year-olds, the prevalence of high myopia was lower than that in Beijing, ${ }^{35}$ Qingdao, ${ }^{36}$ Yiwu and Hong Kong, ${ }^{16,37}$ and similar to that in Mongolia. ${ }^{38}$ In our study, the prevalence of high myopia began to increase between the ages of 11 and 12 years in Qinghai, beginning later than in the developed plains areas of Beijing, ${ }^{35}$ Qingdao, ${ }^{26}$ Yiwu, ${ }^{16}$ and Hong Kong, ${ }^{37}$ but close to the same age in the underdeveloped plains areas of Yunnan ${ }^{39}$ and Mongolia. ${ }^{38}$ Early myopia onset generally leads to rapid and longer duration for myopia progression and, consequently, a higher risk of becoming high myopic later in life.

The prevalence of astigmatism varies according to geographical position and population. The astigmatism prevalence $(26.2 \%, \leq-0.75 \mathrm{D}, 4-12$ years of age) was higher in Yunnan $(12.7 \%, \leq-0.75 \mathrm{D}, 13-14$ years of age $),{ }^{40}$ Beijing $(10 \%, \leq-0.75 \mathrm{D}, 5-15$ years of age $),{ }^{41}$ and Wenzhou $(20.3 \%, \leq-0.75 \mathrm{D}, 6-9$ years of age $){ }^{42}$ Our prevalence was lower than that in other studies, where the prevalence was $42.7 \%$ in Guangzhou $(\leq-0.75$ D, 5-15 years old) and $36.3 \%$ in Shandong $(\leq-0.75$ D, 4 18 years of age). ${ }^{4,43}$ Different studies have reported contradictory findings, and we detected correlations with age, 
region of habitation, sex and myopia refractive error, unlike those reported by Zhao et al, Lin et al and Fotouhi et al. ${ }^{41,42,44}$

Uncorrected/undercorrected refractive error (particularly myopia) is the main reason for UCVA/PVA 20/40 or worse. ${ }^{43,45,46}$ This type of vision problem can be easily solved by prescribing a pair of suitable spectacles. In the present study, only $24.2 \%$ of myopia students wore glasses, and $59.4 \%$ of them did not have accurate spectacles. Our finding was comparable to another study by Chin MP and colleagues, in which $64 \%$ of Chinese children in remote areas did not have appropriate correction. ${ }^{15}$ The wearing glass rate was lower than that in developed provinces of China (70.1\% among those aged 5-18 years). ${ }^{16}$ In Menyuan, many children are "left-behind children" whose parents are too busy to care about their children's eye health. The price of glasses is more expensive, and the inconvenience of wearing glasses may also be the reason for not wearing glasses among myopic children. Another potential reason for glasses nonwear among myopic children may be related to the widespread misunderstanding in China that young children wearing glasses might damage their visual acuity. ${ }^{47}$ The lower appropriate rate of glasses use among Qinghai children may also be associated with poor access to health services. ${ }^{48,49}$

The projected number of children and adolescents 7 to 18 years of age affected by reduced visual acuity is approximately 152 million in 2020 and approximately 180 million in $2030 .^{50}$ Visual impairment affects an individual's quality of life and impedes access to education and employment. ${ }^{51}$ Therefore, in Qinghai, we should adopt certain measures and strategies, such as strengthening children's vision screening, improving the local medical level, popularizing the knowledge of eye care, enhancing the awareness of children and parents of eye care, and providing optician subsidies, to reduce and delay the occurrence of myopia in children and adolescents.

Our study has potential limitations. Children aged 4-6 years are young, and many parents refuse to participate in the eye study because of concerns about pupil dilation. Therefore, relatively few children were included, which may have affected the calculation of the prevalence of refractive error. Additionally, astigmatism was related to sex, place of residence, age, and myopia, but some studies differ from ours, likely because of the different age groups and different criteria for astigmatism definition.

\section{Conclusion}

Refractive error in 4- to 12-year-old children, particularly children with myopia, is common in Northwest Plateau China. In our study, $29.8 \%$ of 4- to 12-year-old children had myopia, $0.9 \%$ had high myopia, $4.3 \%$ had medium to marked hyperopia, and $26.2 \%$ had astigmatism. Uncorrected/undercorrected refractive error (especially myopia) was the main reason for PVA 20/40 or worse. A total of $75.8 \%$ of myopic children did not wear glasses, and $59.4 \%$ of myopic children had inaccurate spectacles. The prevention and treatment of myopia are critical. We should focus more on the eyesight health of children in the northwest plateau region to further explore opportunities and methods to prevent and control myopia.

\section{Data Sharing Statement}

Please contact author for data requests.

\section{Ethics Approval and Consent to Participate}

The ethics committee of Affiliated Eye Hospital of Shandong University of Traditional Chinese Medicine.

\section{Consent for Publication}

The authors consent for publication.

\section{Patient Consent}

Obtained.

\section{Acknowledgments}

The authors would like to acknowledge the support and participation of the Affiliated Eye Hospital of Shandong University of Traditional Chinese Medicine, the Administration of the Education and School Board of Menyuan, the schools, the children, and their parents and guardians in the China Eye Study.

\section{Author Contributions}

All authors made substantial contributions to conception and design, acquisition of data, or analysis and interpretation of data; took part in drafting the article or revising it critically for important intellectual content; agreed to submit to the current journal; gave final approval of the version to be published; and agree to be accountable for all aspects of the work. 


\section{Funding}

This work was supported by National Key Research and Development Project (2019YFC1710200, 2019YFC1 710203); Shandong Medical and health Science and Technology Development Project (2019WS571, 2019WS570). The Natural Science Foundation of Shandong Province (ZR2020QH314).

\section{Disclosure}

The authors declare no conflicts of interest.

\section{References}

1. Tideman JWL, Polling JR, Vingerling JR, et al. Axial length growth and the risk of developing myopia in European children. Acta Ophthalmol. 2018;96(3):301-309. doi:10.1111/aos.13603

2. Bourne RR, Stevens GA, White RA, et al. Causes of vision loss worldwide 1990-2010: a systematic analysis. Lancet Glob Health. 2013;1(6):e339-349. doi:10.1016/S2214-109X(13)70113-X

3. Pan CW, Wu RK, Li J, Zhong H. Low prevalence of myopia among school children in rural China. BMC Ophthalmol. 2018;18(1):140. doi:10.1186/s12886-018-0808-0

4. Wu JF, Bi HS, Wang SM, et al. Refractive error, visual acuity and causes of vision loss in children in Shandong, China. The Shandong Children Eye Study. PLoS One. 2013;8(12):e82763. doi:10.1371/ journal.pone.0082763

5. Czepita M, Czepita D, Safranow K. Role of gender in the prevalence of myopia among polish schoolchildren. $J$ Ophthalmol. 2019;2019:9748576. doi:10.1155/2019/9748576

6. Tideman JW, Polling JR, Voortman T, et al. Low serum vitamin D is associated with axial length and risk of myopia in young children. Eur J Epidemiol. 2016;31(5):491-499. doi:10.1007/s10654-016-0128-8

7. Holden BA, Fricke TR, Wilson DA, et al. Global prevalence of myopia and high myopia and temporal trends from 2000 through 2050. Ophthalmology. 2016;123(5):1036-1042. doi:10.1016/j. ophtha.2016.01.006

8. Hopf S, Pfeiffer N. Epidemiology of myopia. Ophthalmologe. 2017;114(1):20-23. doi:10.1007/s00347-016-0361-2

9. Dong L, Kang YK, Li Y, Wei WB, Jonas JB. prevalence and time trends of myopia in children and adolescents in china: a systemic review and meta-analysis. Retina. 2020;40(3):399-411. doi:10.1097/ IAE.0000000000002590

10. Wong TY, Ferreira A, Hughes R, Carter G, Mitchell P. Epidemiology and disease burden of pathologic myopia and myopic choroidal neovascularization: an evidence-based systematic review. Am J Ophthalmol. 2014;157(1):9-25.e12. doi:10.1016/j. ajo.2013.08.010

11. Ghorbani Mojarrad N, Plotnikov D, Williams C, Guggenheim JA; UK Biobank Eye and Vision Consortium. Association between polygenic risk score and risk of myopia. JAMA Ophthalmol. 2019;138 (1):7-13. doi:10.1001/jamaophthalmol.2019.4421

12. Lingham G, Mackey DA, Lucas R, Yazar S. How does spending time outdoors protect against myopia? A review. $\mathrm{Br} J$ Ophthalmol. 2020;104(5):593-599. doi:10.1136/bjophthalmol-2019-314675

13. Wu PC, Chen CT, Lin KK, et al. Myopia prevention and outdoor light intensity in a school-based cluster randomized trial. Ophthalmology. 2018;125(8):1239-1250. doi:10.1016/j.ophtha.2017.12.011

14. Lin Z, Gao TY, Vasudevan B, et al. Near work, outdoor activity, and myopia in children in rural China: the Handan Offspring Myopia Study. BMC Ophthalmol. 2017;17(1):203. doi:10.1186/s12886-0170598-9
15. Chin MP, Siong KH, Chan KH, Do CW, Chan HH, Cheong AM. Prevalence of visual impairment and refractive errors among different ethnic groups in schoolchildren in Turpan, China. Ophthalmic Physiol Opt. 2015;35(3):263-270. doi:10.1111/opo.12193

16. Wang J, Ying GS, Fu X, et al. Prevalence of myopia and vision impairment in school students in Eastern China. BMC Ophthalmol. 2020;20(1):2. doi:10.1186/s12886-019-1281-0

17. Hu M, Zhou Y, Huang S, et al. Population prevalence of myopia, glasses wear and free glasses acceptance among minority versus Han schoolchildren in China. PLoS One. 2019;14(4):e0215660. doi:10.1371/journal.pone. 0215660

18. Thorn F, Chen J, Li C, et al. Refractive status and prevalence of myopia among Chinese primary school students. Clin Exp Optom. 2020;103(2):177-183. doi:10.1111/cxo.12980

19. Shen Y, Chang C, Zhang J, Jiang Y, Ni B, Wang Y. Prevalence and risk factors associated with hypertension and prehypertension in a working population at high altitude in China: a Cross-Sectional Study. Environ Health Prev Med. 2017;22(1):19. doi:10.1186/ s12199-017-0634-7

20. Riley CJ, Gavin M. Physiological changes to the cardiovascular system at high altitude and its effects on cardiovascular disease. High Alt Med Biol. 2017;18(2):102-113. doi:10.1089/ham.2016.0112

21. Yan X. Cognitive impairments at high altitudes and adaptation. High Alt Med Biol. 2014;15(2):141-145. doi:10.1089/ham.2014.1009

22. Wang J, Zhou Y, Liang Y, Liu Z, Large Sample A. Survey of Tibetan people on the Qinghai-Tibet plateau: current situation of depression and risk factors. Int J Environ Res Public Health. 2019;17(1):289. doi:10.3390/ijerph17010289

23. Jha KN. High altitude and the eye. Asia Pac J Ophthalmol (Phila). 2012;1(3):166-169. doi:10.1097/APO.0b013e318253004e

24. Xie HL, Xie ZK, Ye J, Yang XJ, Qu J. Analysis of correlative factors and prevalence on China's youth myopia. Zhonghua Yi Xue Za Zhi. 2010;90(7):439-442.

25. Dong YH, Liu HB, Wang ZH, Xu RB, Yang ZP, Ma J. The epidemic status and secular trends of myopia prevalence for Chinese children and adolescents aged 7-18 years from 2005 to 2014. Zhonghua Yu Fang Yi Xue Za Zhi. 2017;51(4):285-289.

26. Qian X, Liu B, Wang J, et al. Prevalence of refractive errors in Tibetan adolescents. BMC Ophthalmol. 2018;18(1):118. doi:10.1186/s12886-018-0780-8

27. Ip JM, Huynh SC, Robaei D, et al. Ethnic differences in refraction and ocular biometry in a population-based sample of 11-15-year-old Australian children. Eye (Lond). 2008;22(5):649-656. doi:10.1038/sj. eye. 6702701

28. Terasaki H, Yamashita T, Yoshihara N, Kii Y, Sakamoto T. Association of lifestyle and body structure to ocular axial length in Japanese elementary school children. BMC Ophthalmol. 2017;17 (1):123. doi:10.1186/s12886-017-0519-y

29. $\mathrm{Lu} \mathrm{TL}, \mathrm{Wu} \mathrm{JF}$, Ye X, et al. Axial length and associated factors in children: the Shandong Children Eye Study. Ophthalmologica. 2016;235(2):78-86. doi:10.1159/000441900

30. Xie Z, Long Y, Wang J, Li Q, Zhang Q. Prevalence of myopia and associated risk factors among primary students in Chongqing: multilevel modeling. BMC Ophthalmol. 2020;20(1):146. doi:10.1186/ s12886-020-01410-3

31. Wu H, Chen W, Zhao F, et al. Scleral hypoxia is a target for myopia control. Proc Natl Acad Sci USA. 2018;115(30):E7091-E7100. doi:10.1073/pnas.1721443115

32. He M, Xiang F, Zeng Y, et al. Effect of time spent outdoors at school on the development of myopia among children in China: a randomized clinical trial. JAMA. 2015;314(11):1142-1148. doi:10.1001/jama.2015.10803

33. Ho CL, Wu WF, Liou YM. Dose-response relationship of outdoor exposure and myopia indicators: a systematic review and meta-analysis of various research methods. Int $J$ Environ Res Public Health. 2019;16(14):E2595. doi:10.3390/ijerph16142595 
34. Li Y, Liu J, Qi P. The increasing prevalence of myopia in junior high school students in the Haidian District of Beijing, China: a 10-year population-based survey. BMC Ophthalmol. 2017;17(1):88 . doi:10.1186/s12886-017-0483-6

35. Guo Y, Duan JL, Liu LJ, et al. High myopia in greater Beijing school children in 2016. PLoS One. 2017;12(11):e0187396. doi:10.1371/ journal.pone. 0187396

36. Sun JT, An M, Yan XB, Li GH, Wang DB. Prevalence and related factors for myopia in school-aged children in Qingdao. J Ophthalmol. 2018;2018:9781987. doi:10.1155/2018/9781987

37. Lam CS, Lam CH, Cheng SC, Chan LY. Prevalence of myopia among Hong Kong Chinese schoolchildren: changes over two decades. Ophthalmic Physiol Opt. 2012;32(1):17-24. doi:10.1111/ j.1475-1313.2011.00886.x

38. Guo K, Yang DY, Wang Y, et al. Prevalence of myopia in schoolchildren in Ejina: the Gobi Desert Children Eye Study. Ophthalmol Vis Sci. 2015;56(3):1769-1774. doi:10.1167/iovs.14-15737

39. Qian DJ, Zhong H, Li J, Niu Z, Yuan Y, Pan CW. Myopia among school students in rural China (Yunnan). Ophthalmic Physiol Opt. 2016;36(4):381-387. doi:10.1111/opo.12287

40. Pan CW, Ke C, Hu DN, Li J, Zhong H. Iris colour and astigmatism among Chinese teenagers. Br J Ophthalmol. 2019;103(12):1810-1814.

41. Zhao J, Pan X, Sui R, Munoz SR, Sperduto RD, Ellwein LB. Refractive error study in children: results from Shunyi District, China. Am J Ophthalmol. 2000;129(4):427-435. doi:10.1016/ S0002-9394(99)00452-3

42. Lin Y, Jiang D, Li C, et al. Simultaneous changes in astigmatism with noncycloplegia refraction and ocular biometry in Chinese primary schoolchildren. J Ophthalmol. 2019;2019:5613986. doi:10.1155/ 2019/5613986

43. He M, Zeng J, Liu Y, Xu J, Pokharel GP, Ellwein LB. Refractive error and visual impairment in urban children in southern china. Invest Ophthalmol Vis Sci. 2004;45(3):793-799. doi:10.1167/iovs.03-1051
44. Fotouhi A, Hashemi H, Yekta AA, Mohammad K, Khoob MK Characteristics of astigmatism in a population of schoolchildren, Dezful, Iran. Optom Vis Sci. 2011;88(9):1054-1059. doi:10.1097/ OPX.0b013e318221727d

45. Signes-Soler I, Hernández-Verdejo JL, Estrella Lumeras MA, Tomás Verduras E, Piñero DP. Refractive error study in young subjects: results from a rural area in Paraguay. Int $J$ Ophthalmol. 2017;10 (3):467-472.

46. Signes-Soler I, Piñero DP, Murillo MI, Tablada S. Prevalence of visual impairment and refractive errors in an urban area of Mexico. Int J Ophthalmol. 2019;12(10):1612-1617. doi:10.18240/ijo.2019.10.14

47. Li L, Lam J, Lu Y, et al. Attitudes of students, parents, and teachers toward glasses use in rural China. Arch Ophthalmol. 2010;128 (6):759-765. doi:10.1001/archophthalmol.2010.73

48. Jing F. Health sector reform and reproductive health services in poor rural China. Health Policy Plan. 2004;19(suppl 1):i40-49. doi:10.1093/heapol/czh044

49. Wong HT, Guo YQ, Chiu MY, Chen S, Zhao Y. Spatial illustration of health-care workforce accessibility index in China: how far has our 2009 health-care reform brought us? Aust J Rural Health. 2016;24 (1):54-60. doi:10.1111/ajr.12198

50. Sun HP, Li A, Xu Y, Pan CW. Secular trends of reduced visual acuity from 1985 to 2010 and disease burden projection for 2020 and 2030 among primary and secondary school students in China. JAMA Ophthalmol. 2015;133(3):262-268. doi:10.1001/jamaophthalmol.2014.4899

51. Selvarajah S, Dunt DR, Marella M, et al. Vision impairment and refractive errors in refugees presenting to community optometry clinics in Victoria, Australia. Clin Exp Optom. 2020;103 (5):668-674. doi:10.1111/cxo.13010
International Journal of General Medicine

\section{Publish your work in this journal}

The International Journal of General Medicine is an international, peer-reviewed open-access journal that focuses on general and internal medicine, pathogenesis, epidemiology, diagnosis, monitoring and treatment protocols. The journal is characterized by the rapid reporting of reviews, original research and clinical studies

\section{Dovepress}

across all disease areas. The manuscript management system is completely online and includes a very quick and fair peer-review system, which is all easy to use. Visit http://www.dovepress.com/ testimonials.php to read real quotes from published authors. 